\title{
Genome-wide association study identifies two loci influencing plasma neurofilament light levels
}

Jie-Qiong $\mathrm{Li}^{1 \dagger}$, Xiang-Zhen Yuan ${ }^{1 \dagger}$, Hai-Yan Li ${ }^{2}$, Xi-Peng Cao ${ }^{3}$, Jin-Tai Yu ${ }^{1,3^{*}}$, Lan Tan ${ }^{1,3^{*}}$, Wei-An Chen ${ }^{4^{*}}$ and Alzheimer's Disease Neuroimaging Initiative

\begin{abstract}
Background: Plasma neurofilament light (NFL) is a promising biomarker for Alzheimer disease (AD), which increases in the early stage of $A D$ and is associated with the progression of $A D$. We performed a genome-wide association study (GWAS) of plasma NFL in Alzheimer's Disease Neuroimaging Initiative 1 (ADNI-1) cohort to identify novel variants associated with AD.
\end{abstract}

Methods: This study included 179 cognitively healthy controls (HC), 176 patients with mild cognitive impairment (MCl), and 172 patients with AD. All subjects were restricted to non-Hispanic Caucasian derived from the ADNI cohort and met all quality control (QC) criteria. Association of plasma NFL with the genetic variants was assessed using PLINK with an additive genetic model, i.e.dose-dependent effect of the minor alleles. The influence of a genetic variant associated with plasma NFL (rs7943454) on brain structure was further assessed using PLINK with a linear regression model.

Results: The minor allele (T) of rs7943454 in leucine zipper protein 2 gene (LUZP2) was associated with higher plasma NFL at suggestive levels $\left(P=1.39 \times 10^{-6}\right)$ in a dose-dependent fashion. In contrast, the minor allele $(G)$ of rs640476 near GABRB2 was associated with lower plasma NFL at suggestive levels $\left(P=6.71 \times 10^{-6}\right)$ in a dose-dependent effect in all diagnostic groups except the MCI group. Furthermore, the minor allele (T) of rs7943454 within LUZP2 increased the onset risk of $\mathrm{AD}$ (odds ratio $=1.547$, confidence interval $95 \%=1.018-2.351$ ) and was associated with atrophy of right middle temporal gyrus in the whole cohort in the longitudinal study $(P=0.0234)$.

Conclusion: GWAS found the associations of two single nucleotide polymorphisms (rs7943454 and rs640476) with plasma NFL at suggestive levels. Rs7943454 in LUZP2 was associated with the onset risk of AD and atrophy of right middle temporal gyrusin the whole cohort. Using an endophenotype-based approach, we identified rs7943454 as a new AD risk locus.

Keywords: Genome-wide association study, Plasma NFL, Alzheimer disease, LUZP2, GABRB2, Genetic factors

\footnotetext{
*Correspondence: yu-jintai@163.com; dr.tanlan@163.com; wzanan@126.com Data used in preparation for this article were obtained from the Alzheimer's Disease Neuroimaging Initiative (ADNI) database (adni.loni.usc.edu). As such, the investigators within the ADNI contributed to the design and implementation of ADNI and/or provided data but did not participate in the analysis or writing of this report. A complete listing of ADNI investigators can be found at:http://adni.loni.usc.edu/wp-content/uploads/how_to_apply/

ADNI_Acknowledgement_List.pdf.

${ }^{\dagger}$ Equal contributors

'Department of Neurology, Qingdao Municipal Hospital, Qingdao University, No.5 Donghai Middle Road, Qingdao 266071, Shandong Province, China ${ }^{4}$ Department of Neurology, The First Affiliated Hospital of Wenzhou Medical University, Nanbaixiang Road, Wenzhou 325000, Zhejiang Province, China Full list of author information is available at the end of the article
}

(c) The Author(s). 2018 Open Access This article is distributed under the terms of the Creative Commons Attribution 4.0 International License (http://creativecommons.org/licenses/by/4.0/), which permits unrestricted use, distribution, and reproduction in any medium, provided you give appropriate credit to the original author(s) and the source, provide a link to the Creative Commons license, and indicate if changes were made. The Creative Commons Public Domain Dedication waiver (http://creativecommons.org/publicdomain/zero/1.0/) applies to the data made available in this article, unless otherwise stated. 


\section{Background}

Alzheimer disease (AD) is the main cause of dementia and one of the major challenges for health care across the world, which is characterized pathologically by extracellular accumulation of amyloid- $\beta(A \beta)$, intracellular deposition of neurofibrillary tangles (NFT), neuronal loss and synaptic dysfunction [1]. Well-established cerebrospinal fluid (CSF) biomarkers including $A \beta_{42}$, total-tau (t-tau), phosphorylated tau ( $\mathrm{p}$-tau) have been used for the diagnosis of $\mathrm{AD}$ and monitoring its progression [2], but the their application is hampered by a high degree of invasiveness, complex operations and high costs. Biomarkers in peripheral blood are more appropriate screening tools for AD among old individuals to monitor $\mathrm{AD}$ progression. Interestingly, recent studies using ultrasensitive assay showed that plasma neurofilament light (NFL), the main component of neurofilaments (cytoskeletal protein of neurons), increased in patients with AD dementia and was associated with other established CSF and neuroimaging biomarkers of $\mathrm{AD}[3,4]$. Plasma NFL is a noninvasive biomarker for neuronal injury in $\mathrm{AD}$ compared with CSF biomarkers. Thus, it has the potential for monitoring AD progression [3].

$\mathrm{AD}$ is a clinically heterogeneous neurodegenerative disease with a strong genetic component. Genetic risk factors of $\mathrm{AD}$ impact the CSF or neuroimaging biomarkers through which they might modulate the process of $\mathrm{AD}$ [5]. Thus, biomarkers for $\mathrm{AD}$ may be used as endophenotypes to explore the genetic factors that impact their metabolism [6-8]. Based on the association between plasma NFL and AD, we performed a genomewide association study (GWAS) using plasma NFL as an endophenotype of $\mathrm{AD}$ to explore genetic factors involved in plasma NFL metabolism. We hypothesized that these genetic factors may influence pathological change in AD.

\section{Methods}

\section{Subjects}

In this study, $172 \mathrm{AD}$ patients, 176 subjects with mild cognitive impairment (MCI), and 179 healthy controls (HC) whose data met all quality control (QC) criteria were included from the Alzheimer's Disease Neuroimaging Initiative
1 (ADNI-1) cohort. The full cohort with plasma NFL and genotype data included 578 subjects. All the subjects were restricted to non-Hispanic Caucasian checked with their pedigree information checked to reduce potential bias of population stratification that might confound GWAS results. This step removed 40 subjects. After QC of the plasma NFL levels and removal of 11 outliers, there were 527 subjects with plasma NFL data left. The detailed demographic information and plasma NFL data have been shown in Table 1 .

\section{ADNI dataset}

ADNI was launched in 2003 by the National Institute on Aging, the National Institute of Biomedical Imaging and Bioengineering, the Food and Drug Administration, private pharmaceutical companies and nonprofit organizations. ADNI was established to develop serial magnetic resonance imaging (MRI), positron emission tomography (PET), and a combination of biomarkers, neuropsychological and clinical assessment to improve early diagnosis and measure the progression of $\mathrm{AD}$ [9]. The ADNI database has three protocols (ADNI 1, ADNI 2 and ADNI Grand Opportunities (ADNI GO)) at present and recruited more than 1500 participants including normal older subjects, MCI and early AD in this research. More information is available on the website of ADNI (www. loni.ucla.edu/ADNI).

\section{Plasma measurements and quality control}

Plasma NFL was analyzed using the ultrasensitive Single Molecule array (Simoa) technique as previously described [10]. The assay used a combination of monoclonal antibodies and purified bovine NFL as a calibrator. Analytical sensitivity was $<1.0 \mathrm{pg} / \mathrm{mL}$, and the NFL levels in all tested sample were above the detection limit. Further QC was performed to reduce the potential influence of extreme outliers on statistical results. Mean and standard deviations (SD) of baseline plasma NFL were calculated. Subjects who had a value which is 3 -fold SD greater or smaller than the mean value $(<42.8-3 \times 26.8 \mathrm{pg} / \mathrm{mL}$ or $>$ $42.8+3 \times 26.8 \mathrm{pg} / \mathrm{mL}$ ) were removed from the analysis. This step removed 11 subjects.

Table 1 The demographic information of participants with plasma NFL data

\begin{tabular}{lllll}
\hline Baseline diagnosis & $\mathrm{AD}$ & $\mathrm{MCl}$ & $\mathrm{HC}$ & Total \\
\hline $\mathrm{n}$ & 172 & 176 & 179 & 527 \\
Age (years), mean \pm SD (range) & $76 \pm 7(56-91)$ & $75 \pm 8(54-89)$ & $76 \pm 5(62-90)$ & $75 \pm 7(54-91)$ \\
Gender, male/female & $90 / 82$ & $117 / 59$ & $103 / 76$ & $310 / 217$ \\
APOE \&4 carrier (\%) & 66.9 & 52.8 & 26.8 & 48.6 \\
Plasma NFL (pg/ml), mean \pm SD $^{\text {a }}$ & $48.7 \pm 20.9$ & $39.9 \pm 17.7$ & $32.8 \pm 15.5$ & $40.4 \pm 19.3$ \\
\hline
\end{tabular}

Abbreviations: $A D$ Alzheimer's disease, $A P O E$ Apolipoprotein $\mathrm{E} ; H C$ healthy control, $M C I$ mild cognitive impairment, $N F L$ neurofilament light, $S D$ standard deviation aPlasma NFL levels were different across the 3 diagnostic groups $(P<0.0001)$. Tukey's multiple comparisons test showed that AD patients had higher plasma NFL levels compared with $\mathrm{MCl}$ group and healthy controls $(P<0.001)$. $\mathrm{MCl}$ group also had higher plasma NFL levels compared to healthy controls $(P=0.0007)$ 


\section{Genotyping and quality control}

The ADNI 1 samples involved in this study were genotyped by Human 610-Quad BeadChip (Illumina, Inc., San Diego, CA). PLINK software (version 1.07) was used to explore the association of plasma NFL with the genetic variants using the following stringent criteria: minimum call rate for single nucleotide polymorphisms (SNPs) and individuals > 98\%; minimum minor allele frequencies $(\mathrm{MAF})>0.20$, Hardy-Weinberg equilibrium test $P>0.001$. The restriction to SNPs with a minor allele frequency $>0.20$ served to reduce the potential for false-positive results and enhance statistical power. An apolipoprotein $\mathrm{E}(A P O E)$ genotyping kit was used to identify $A P O E$ alleles, which were defined by rs7412 and rs429358 [7].

\section{Brain structure on MRI}

The data of MRI brain structure were derived from UCSF FreeSurfer datasets, which were used to conduct association test of rs7943454in leucine zipper protein2 gene (LUZP2) with brain structure. The cerebral image segmentation and analysis were performed with the FreeSurfer version5.1 (http://surfer.nmr.mgh.harvard.edu/) based on the2010 Desikan-Killanyatlas [11]. The technical details of these procedures have been described in prior publications [12]. Brian regions have been reported to be closely associated with $\mathrm{AD}$ such as hippocampus, parahippocampus, middle temporal gyrus, posterior cingulate, precuneus and entorhinal cortex, which were selected as our regions of interest (ROI) to analyze their its associations with rs7943454.

\section{Statistical analyses}

Association studies of plasma NFL with the genetic variants were performed using PLINK (version 1.07) with the additive genetic model, i.e. dose-dependent effect of the minor allele. The analysis included a total of 30,1687 genotyped variants. To adjust for multiple testing, Bonferroni correction was applied and SNPs with corrected $p<0.01$ (uncorrected $p<3.31 \times 10-8$, i.e., $0.01 / 301687$ markers) were considered genome-wide significant. And we secondarily examined SNPs with uncorrected $p$ values less than 10-5 to identify potential candidates. Age, gender and diagnosis were included as covariates. Bonferroni correction of the $P$ values by the total number of acceptable quality SNPs was used for multiple test correction. Differences in continuous variables (plasma NFL levels, volume of regional brain) were examined using one-way analysis of variance (ANOVA), and Tukey's multiple comparisons test was used to perform the pairwise analysis after ANOVA. Genome-wide associations were visualized using a software program ( $\mathrm{R}$, version 3.4.0; The $\mathrm{R}$ Foundation). Regional associations were visualized with the Locus Zoom web tool (http://locuszoom.org/). Moreover, a multiple linear regression model was applied using PLINK to estimate coefficients for testing a possible correlation between brain structure and rs7943454. Age, gender, education, and APOE $\varepsilon 4$ status were used as covariates.

\section{Results}

\section{Characteristics of included subjects}

The information about these included subjects has been shown in Table 1. Briefly, 172 AD (82 women, $76 \pm 7$ years), $176 \mathrm{MCI}$ ( 59 women, $75 \pm 8$ years) and $179 \mathrm{HC}$ (76 women, $76 \pm 5$ years) subjects were recruited in this study. AD group had the highest frequency of $\varepsilon 4$ allele within $A P O E$ gene $(66.9 \%)$. AD patients $(48.7 \pm 20.9 \mathrm{pg} / \mathrm{ml})$ had higher plasma NFL levels compared with MCI group $(39.9 \pm 17.7 \mathrm{pg} / \mathrm{ml})$ and $\mathrm{HC}$ group $(32.8 \pm 15.5 \mathrm{pg} / \mathrm{ml})$ $(P<0.001)$. MCI group also had higher plasma NFL levels compared to $\mathrm{HC}$ group $(P=0.0007)$. The sensitivity and specificity of plasma NFL used for the diagnosis of $\mathrm{AD}$ were 0.73 and 0.84 respectively. The area under the curve (AUC) of the model containing plasma NFL, age at baseline, gender, educational level and APOE $\varepsilon 4$ genotype was 0.86 in predicting the onset of $\mathrm{AD}$ among $\mathrm{HC}$ controls group. By comparison, the AUROCs were 0.84 to 0.87 for CSF A $\beta 42$, CSF ttau, and CSF p-tau (Additional file 1: Figure S1).

\section{SNPs associated with plasma NFL levels}

There were 527 individuals with plasma NFL data as mentioned above. After adjusting for age, gender and diagnosis, two SNPs (rs7943454, rs640476) were identified associated with plasma NFL at suggestive levels of $P<10^{-5}$ (Fig. 1a, Table 2). No SNPs with genome-wide significant association with plasma NFL levels was identified in this study.

The minor allele of rs7943454 (T) was associated with higher plasma NFL levels in a dose-dependent effect in all diagnostic groups (Fig. 2a). In contrast, the minor allele of rs640476 (G) showed association with lower plasma NFL levels in a dose-dependent effect in all diagnostic groups except the MCI group (Fig. 2b).

SNPs mapped closely to the two suggestive SNPs (rs7943454, rs640476) regions were also analyzed. These nearby SNPs showed association with plasma NFL levels at $P$ levels lower than 0.01 . However, these SNPs associated with plasma NFL also disappeared after controlling the genotype of the two suggestive SNPs (Fig. 1b and c, Fig. $1 \mathrm{~d}$ and e). The results indicated that these nearby SNPs were driven by the two suggestive SNPs.

\section{Rs7943454 and onset risk of AD}

The International Genomics of Alzheimer's Disease Project (IGAP) is the largest genetic epidemiology investigation of AD risk to date. In 2013, the IGAP reported a grandscale meta-analysis and identified 11 new susceptibility loci 

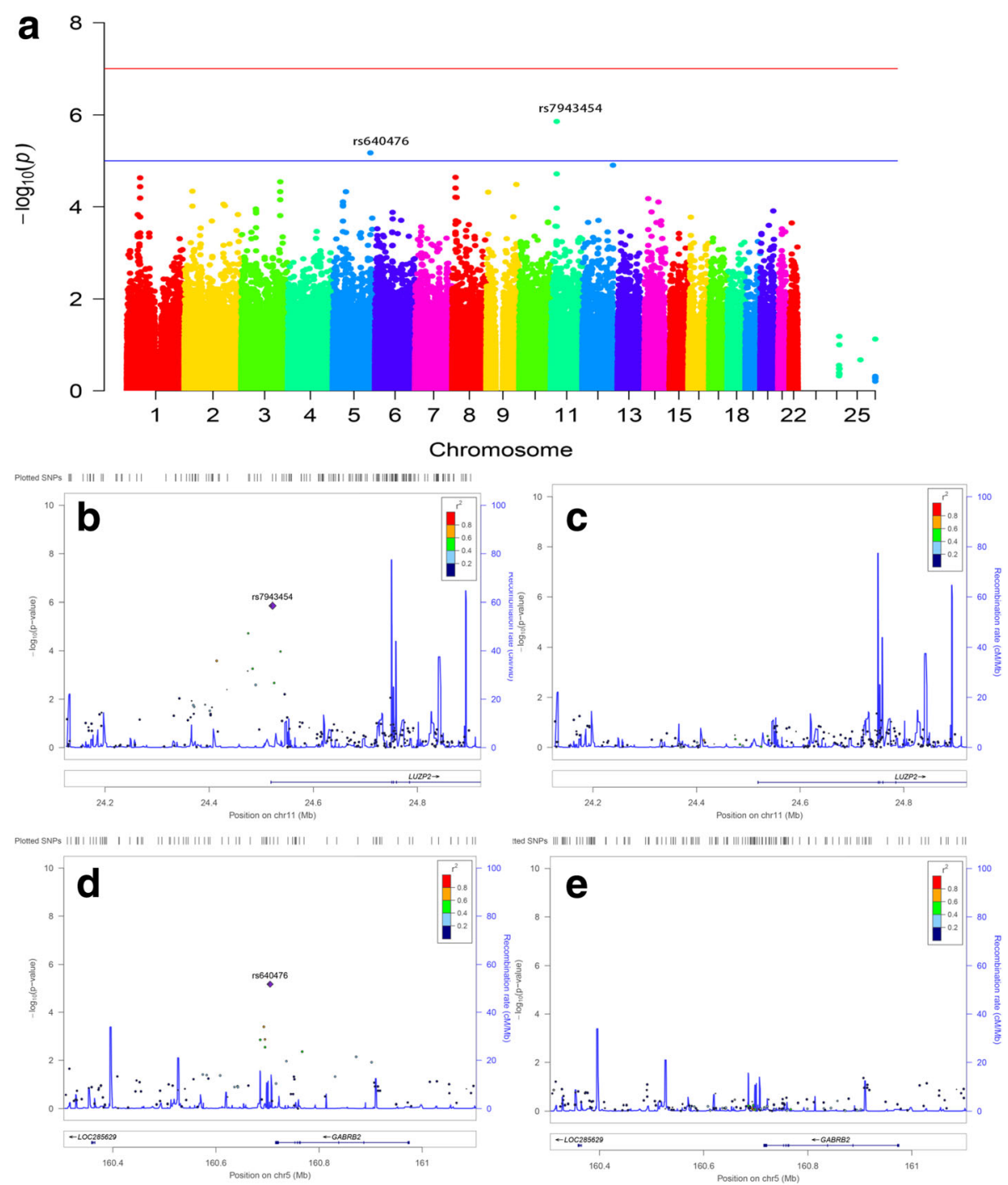

Fig. 1 Manhattan and regional plots for associations with plasma NFL levels. a Genome-wide signal intensity (Manhattan) plots showing the $\log _{10}(p$-value) for individual SNPs. b Regional association results for the $24.2 \mathrm{Mb}$ to $24.8 \mathrm{Mb}$ region of chromosome 11 . c Association results for $24.2 \mathrm{Mb}$ to $24.8 \mathrm{Mb}$ region of chromosome 11 controlling for rs7943454. d Regional association results for the $160.4 \mathrm{Mb}$ to $161 \mathrm{Mb}$ regions of chromosome 5. e Association results for $160.4 \mathrm{Mb}$ to $161 \mathrm{Mb}$ regions of chromosome 5 controlling for rs640476

for AD [13]. The IGAP research was divided into a discovery step (stage 1 ) and a replication step (stage 2). We checked the two loci associated with plasma NFL in IGAP database in the stage 1 meta-analysis and identified rs7943454 as a risk locus for $\mathrm{AD}(P=0.03476)$.

Table 2 Top SNPs associated with plasma NFL levels $\left(P\right.$ values $\left.<10^{-5}\right)$

\begin{tabular}{llllll}
\hline CHR & SNP & MAF & Closest Gene & SNP Type/Location & $P$ values \\
\hline 11 & rs7943454 & 0.460 & LUZP2 & intron & $1.39 \times 10^{-6}$ \\
5 & rs640476 & 0.297 & GABRB2 & intergenic & $6.71 \times 10^{-6}$
\end{tabular}

Abbreviations: CHR chromosome, LUZP2 leucine zipper protein 2, MAF minor allele frequency, SNP single nucleotide polymorphism, GABRB2 gammaaminobutyric acid type $A$ receptor beta2 subunit
The minor allele of rs7943454 (T) increased the onset risk of AD 1.547-fold in our analysis (odds ratio $=1.547$, confidence interval $95 \%=1.018-2.351)$.

\section{Impact of rs7943454 on brain structure}

Several cortical areas including middle temporal gyrus, posterior cingulate, precuneus, parahippocampal gyrus, and hippocampus were chosen as the $\mathrm{ROI}$ of the $\mathrm{AD}$ related MRI measures analysis. We analyzed the association of rs7943454 with $\mathrm{AD}$ related brain structures in a linear model using age, gender, education years, $A P O E \varepsilon 4$ status and intracranial volume (ICV) as covariates. There was no regional cortical volume associated with rs7943454 at baseline in the hybrid population (AD, MCI, and $\mathrm{HC}$ subjects) 


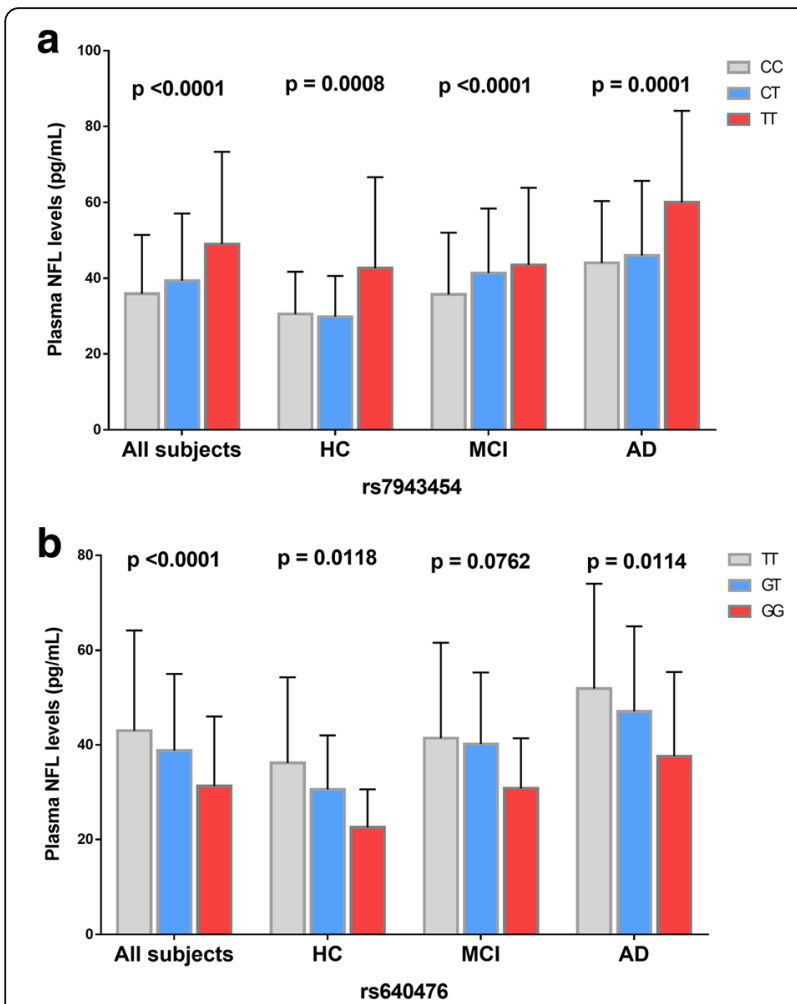

Fig. 2 Mean plasma NFL levels of different diagnostic groups and genotypes. Mean and standard errors of plasma NFL levels are shown for groups defined by baseline diagnosis and genotypes. $P<0.05$ was considered statistically significant after examination with a multiple linear regression model using age, gender and the diagnosis as covariates. a The minor alleleofrs7943454 (T) showed association with higher plasma NFL levels in a dose-dependent effect in all diagnostic groups. b The minor allele of rs640476 (G) showed association with lower plasma NFL levels in a dosedependent effect in all diagnostic groups except the $\mathrm{MCl}$ group

(Fig. 3a). However, rs7943454 increased the percentage of atrophy of right middle temporal gyrus in the hybrid population in the one-year follow-up research $(P=0.0234$, Fig. 3b). Subjects with TT genotype had greater atrophy rate of right middle temporal gyrus than those with CC genotype (CC: $0.9880 \pm 0.0622$, CT: $0.9783 \pm 0.0377$, TT: 0.9537 $\pm 0.0433)(P=0.05)$. There was no significant difference in the volume of right middle temporal gyrus between CT genotype and $\mathrm{CC}$ genotype or TT genotype.

\section{Discussion}

To our knowledge, this study was the first one using plasma NFL as an endophenotype of AD for GWAS. The use of quantitative traits in GWAS has been shown to increase statistical power over case-control designs [8]. We identified two SNPs (rs7943454, rs640476) associated with plasma NFL at suggestive levels. The minor allele (T) of rs7943454 within LUZP2 increased the onset risk of $\mathrm{AD}$ and was associated with atrophy of right middle temporal gyrus in the entire cohort of the one-

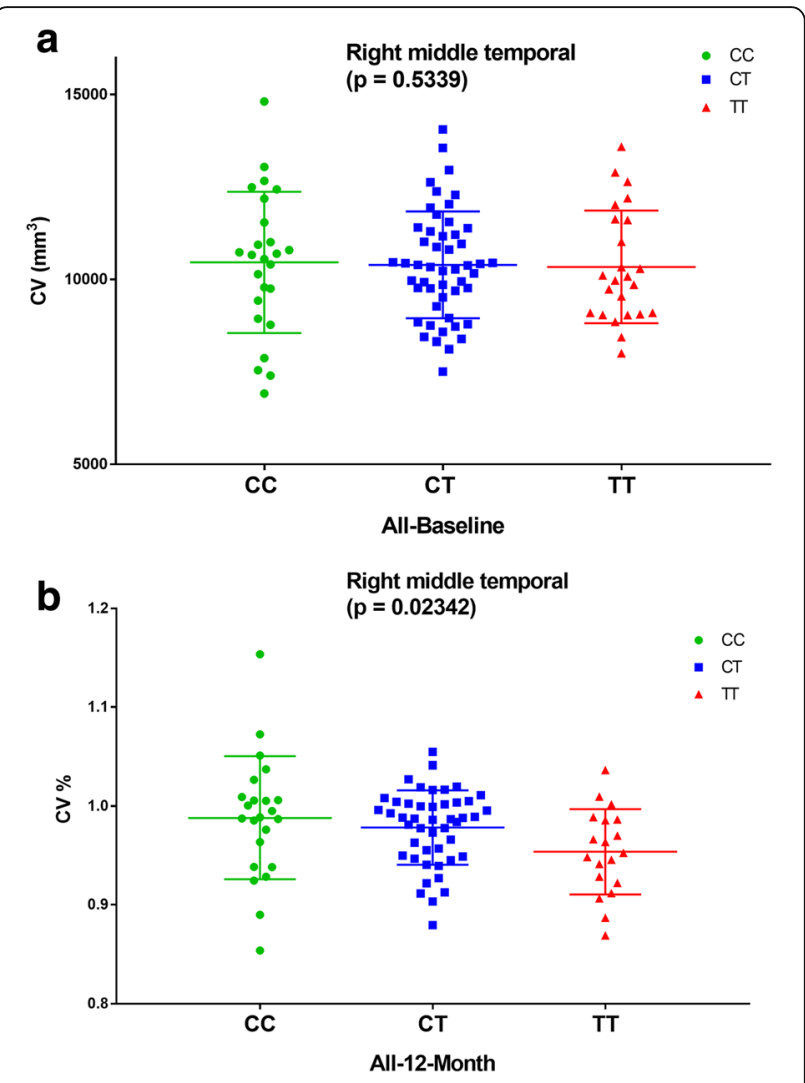

Fig. 3 Rs7943454 and right middle temporal gyrus. a The volume of right middle temporal gyrus was not associated with rs7943454 at baseline in the whole cohort $(P=0.5339)$. b Rs7943454 increase the percentage of atrophy of right middle temporal gyrus in the whole cohort in the following-up research of 1 year $(P=0.0234)$. Subjects with $\Pi$ genotypes had greater atrophy rate of right middle temporal gyrus than those with CC genotypes $(P=0.05)$

year longitudinal study. The use of plasma NFL as an endophenotype of AD for GWAS enabled us to identify a novel $\mathrm{AD}$ candidate gene in addition to examining the influence of well-known $\mathrm{AD}$ genes on $\mathrm{AD}$ biomarkers. We also found the minor allele (T) of rs7943454 in LUZP2 was associated with higher plasma NFL levels in a dose-dependent fashion. In contrast, the minor allele (G) of rs640476 near GABRB2 was associated with lower plasma NFL at suggestive levels in a dose-dependent effect in all diagnostic groups except the MCI group.

Plasma NFL showed significant increase in AD patients than in MCI and healthy controls $(P<0.001)$. Similar with CSF NFL, plasma is not disease-specific and even more marked increases are found in several other neurodegenerative disorders. Increasing evidence indicates that plasma NFL is a potential biomarker for the progression of $\mathrm{AD}$ but not for the diagnosis [4, 14-16]. Plasma NFL is noninvasive and has diagnostic accuracy for $\mathrm{AD}$ in the same range as established CSF biomarkers [3]. Plasma NFL may be widely used as a biomarker in 
clinical studies and drug development of AD, although there is still a long way to go. The elevated NFL levels in plasma also indicate that the degeneration of largecaliber axons plays an important role in the progression of $\mathrm{AD}$ [14].

The variation rs7943454 is located on chromosome 11 p14.3 within $L U Z P 2$ region. $L U Z P 2$ is a leucine zipper protein coding gene that has been reported to be deleted in some patients with Wilms tumor-Aniridia-Genitourinary anomalies-mental Retardation (WAGR) syndrome [17]. It has also been reported that LUZP2 was associated with prostate cancer and hypereosinophilic syndrome [18, 19]. The function of $L U Z P 2$ is still unclear and inhibition of its expression did not show any obvious abnormal phenotypes in mice [20]. Rs7943454 in LUZP2 has been reported as a risk locus of $\mathrm{AD}$ in IGAP, which has been validated in our analysis. The minor allele (T) of rs7943454 increased plasma NFL levels in a dose-dependent fashion and was associated with atrophy of right middle temporal gyrus in the hybrid population. Neuroimaging changes occur years before cognitive decline and middle temporal gyrusis identified as a critical region of memory that changes in the early stage of $\mathrm{AD}$, followed by progressive neocortical damage. Atrophy of middle temporal gyrus is now considered to be a valid diagnostic marker in the early stage of $\mathrm{AD}$ [21]. Our study showed that the minor allele (T) of rs7943454 increased the atrophy rate of right middle temporal gyrus in the one-year follow-up study and the $\mathrm{C}$-allele remarkably prevented its atrophy. This result further proved that rs7943454 was associated with the onset risk of $\mathrm{AD}$ and LUZP2 may be a new susceptibility gene of $\mathrm{AD}$. However, we didn't find any relation between rs7943454 and right middle temporal gyrus in subgroups due to the limited sample size.

The variation rs640476 is located on chromosome 5 $\mathrm{q} 34$, the intergenic region between GABRB2 (gammaaminobutyric acid type A receptor beta2 subunit) and LOC285629 (also known as LINC02159, long intergenic non-protein coding RNA 2159). GABRB2 is the gene coding $\beta 2$ subunit of $\gamma$-aminobutyric acid receptor type A (GABAA receptor), which is the major mediator of fast inhibitory synaptic transmission in the central nervous system. Mutations in GABRB2 genes have been reported to be associated with intellectual disability and epilepsy [22, 23]. A missense mutation in GABRB2 was also reported to be associated with early myoclonic encephalopathy [24]. Mutations in GABRB2 may reduce the expression of GABAA receptor and change the channel function, which could perturb GABA ergic inhibition in the brain. Disruption of excitatory-inhibitory (E/I) balance may be an important mechanism contributing to $\mathrm{AD}$ cognitive decline. Interestingly, despite vast neuronal loss in AD patients, GABA ergic neurons and receptors are relatively spared [25]. We have conducted a linkage disequilibrium analysis in order to annotate the identified functional variants. We found five loci which has strong linkage disequilibrium with rs7943454 (rs1509601: $r^{2}=0.81, \mathrm{D}^{\prime}=1$; rs7927899: $r^{2}=0.81, \mathrm{D}^{\prime}=1$; rs6484052: $r^{2}=0.99, \mathrm{D}^{\prime}=1$; rs6484053: $r^{2}=0.8, \mathrm{D}^{\prime}=0.98$; rs4922682: $r^{2}=0.91, \mathrm{D}^{\prime}=0.96$ ). All these loci are also located in an intron region. We detected only one locus which has strong linkage disequilibrium with rs640476 (rs587875: $\left.r^{2}=0.98, \mathrm{D}^{\prime}=1\right)$ and was also in an intergenic region.

Replication studies with independent, larger samples will be important to confirm these findings. In this study, we used a stringent MAF threshold $(\mathrm{MAF}>0.20$ ) and stringent Bonferroni corrections. These restrictions can improve statistical power to avoid false positive result but may miss less common SNPs. The modest number of subjects restricts stratified analyses for the three diagnostic groups. Besides, a two-year follow-up may be too short to observe the influence of rs7943454 on brain structure changes.

\section{Conclusions}

In summary, we identified that two SNPs (rs7943454 in $L U Z P 2$ and rs640476 near GABRB2) were associated with plasma NFL at suggestive levels. Rs7943454 in LUZP2 was associated with the onset risk of $\mathrm{AD}$ and atrophy of right middle temporal gyrus in the whole cohort. Using endophenotype-based approach, we identified rs7943454 as a new $\mathrm{AD}$ risk locus.

\section{Additional file}

Additional file 1: Figure S1. Plasma neurofilament light for $A D$ diagnosis. Receiver operating cuves of logistic regression model are controlled for age at baseline, gender, educational level and APOE $\varepsilon 4$ genotype. (PDF 6 kb)

\section{Abbreviations}

AD: Alzheimer disease; ADNI: Alzheimer's Disease Neuroimaging Initiative; ANOVA: One-way analysis of variance; APOE: Apolipoprotein $E_{;} A \beta$ : amyloid- $\beta$; CSF: Cerebrospinal fluid; GABRB2: Gamma-aminobutyric acid type A receptor beta2 subunit; GWAS: Genome-wide association study; ICV: Intracranial volume; IGAP: The International Genomics of Alzheimer's Disease Project; LUZP2: Leucine zipper protein 2 gene; MAF: Minimum minor allele frequencies; MCl: Mild cognitive impairment; MRI: Magnetic resonance imaging; NFL: Neurofilament light; NFT: Neurofibrillary tangles; PET: Positron emission tomography; p-tau: Phosphorylated tau; t-tau: Total-tau; QC: Quality control; ROI: Regions of interest; SD: Standard deviations; SNPs: Single nucleotide polymorphisms; WAGR: Wilms tumor-Aniridia-Genitourinary anomalies-mental Retardation

\section{Acknowledgements}

The authors thank scientists contributed in developing the clinical and genetic resources necessary to collect these data and complete this project. The authors also gratefully thank the efforts of hundreds of individuals whose help and participation made this work possible. Data were obtained from the Alzheimer's Disease Neuroimaging Initiative (ADNI) database (adni.loni.usc.edu). 


\section{Funding}

Data collection and sharing for this project was funded by the Alzheimer's Disease Neuroimaging Initiative (ADNI) (National Institutes of Health Grant U01 AG024904) and DOD ADNI (Department of Defense award number W81XWH-12-2-0012). ADNI is funded by the National Institute on Aging, the National Institute of Biomedical Imaging and Bioengineering, and through generous contributions from the following: AbbVie, Alzheimer's Association: Alzheimer's Drug Discovery Foundation; Araclon Biotech; BioClinica, Inc.; Biogen; Bristol-Myers Squibb Company; CereSpir, Inc.; Cogstate; Eisai Inc.; Elan Pharmaceuticals, Inc:; Eli Lilly and Company; Eurolmmun; F. Hoffmann-La Roche Ltd. and its affiliated company Genentech, Inc.; Fujirebio; GE Healthcare; IXICO Ltd.; Janssen Alzheimer Immunotherapy Research \& Development, LLC; Johnson \& Johnson Pharmaceutical Research \& Development LLC.; Lumosity; Lundbeck; Merck \& Co., Inc; Meso Scale Diagnostics, LLC.; NeuroRx Research; Neurotrack Technologies; Novartis Pharmaceuticals Corporation; Pfizer Inc. Piramal Imaging; Servier; Takeda Pharmaceutical Company; and Transition Therapeutics. The Canadian Institutes of Health Research is providing funds to support ADNI clinical sites in Canada. Private sector contributions are facilitated by the Foundation for the National Institutes of Health (www.fnih.org). The grantee organization is the Northern California Institute for Research and Education, and the study is coordinated by the Alzheimer's Therapeutic Research Institute at the University of Southern California. ADNI data are disseminated by the Laboratory for Neuro Imaging at the University of Southern California. This work was supported by grants from the Taishan Scholars Program of Shandong Province (ts201511109 and tsqn20161079), Qingdao Key Health Discipline Development Fund, Qingdao Outstanding Health Professional Development Fund, and Qingdao Innovation and Entrepreneurship Leading Talent Program.

\section{Availability of data and materials}

Data are available to researchers by applying to the respective organizations, ADNI and IGAP. The ADNI data are available at (http://adni.loni.usc.edu/). The IGAP data are available at (http://web.pasteur-lille.fr/en/recherche/u744/igap/ igap_download.php).

\section{Authors' contributions}

JQL, XZY, LT, WAC and JTY: design and conceptualization, analysis and interpretation of the data, drafting and revision of the manuscript. HYL: experimental implementation, data collection and analysis. JQL: experimental implementation, data analysis and revision of the manuscript. XPC: revision of the manuscript. All authors have read and approved the manuscript.

\section{Ethics approval and consent to participate}

Regional ethical committees of all participating institutions approved the ADNI (https://adni.loni.usc.edu/wp-content/uploads/how_to_apply/ ADNI_Acknowledgement_List.pdf). All study participants or authorized representatives provided written informed consent. Ethics approval was obtained from the institutional review boards of each institution involved: Oregon Health and Science University; University of Southern California; University of California—San Diego; University of Michigan; Mayo Clinic, Rochester; Baylor College of Medicine; Columbia University Medical Center; Washington University, St. Louis; University of Alabama at Birmingham; Mount Sinai School of Medicine; Rush University Medical Center; Wien Center; Johns Hopkins University; New York University; Duke University Medical Center; University of Pennsylvania; University of Kentucky; University of Pittsburgh; University of Rochester Medical Center; University of California, Irvine; University of Texas Southwestern Medical School; Emory University; University of Kansas, Medical Center; University of California, Los Angeles; Mayo Clinic, Jacksonville; Indiana University; Yale University School of Medicine; McGill University, Montreal-Jewish General Hospital; Sunnybrook Health Sciences, Ontario; U.B.C.Clinic for AD \& Related Disorders; Cognitive Neurology — St. Joseph's, Ontario; Cleveland Clinic Lou Ruvo Center for Brain Health; Northwestern University; Premiere Research Inst (Palm Beach Neurology); Georgetown University Medical Center; Brigham and Women's Hospital; Stanford University; Banner Sun Health Research Institute; Boston University; Howard University; Case Western Reserve University; University of California, Davis-Sacramento; Neurological Care of CNY; Parkwood Hospital; University of Wisconsin; University of California, Irvine—BIC; Banner Alzheimer's Institute; Dent Neurologic Institute; Ohio State University; Albany Medical College; Hartford Hospital, Olin Neuropsychiatry Research Center; Dartmouth-Hitchcock Medical Center; Wake Forest University Health
Sciences; Rhode Island Hospital; Butler Hospital; UC San Francisco; Medical University South Carolina; St. Joseph's Health Care Nathan Kline Institute; University of lowa College of Medicine; Cornell University; and University of South Florida: USF Health Byrd Alzheimer's Institute.

\section{Competing interests}

The authors declare that they have no competing interests.

\section{Publisher's Note}

Springer Nature remains neutral with regard to jurisdictional claims in published maps and institutional affiliations.

\section{Author details \\ 1Department of Neurology, Qingdao Municipal Hospital, Qingdao University, No.5 Donghai Middle Road, Qingdao 266071, Shandong Province, China. ${ }^{2}$ Department of Neurology, Weihaiwei People's Hospital, Weihai, China. ${ }^{3}$ Clinical Research Center, Qingdao Municipal Hospital, Qingdao University, Qingdao, China. ${ }^{4}$ Department of Neurology, The First Affiliated Hospital of Wenzhou Medical University, Nanbaixiang Road, Wenzhou 325000, Zhejiang Province, China.}

Received: 4 January 2018 Accepted: 1 May 2018

Published online: 10 May 2018

\section{References}

1. Alzheimer's Assoc. 2015 Alzheimer's disease facts and figures. Alzheimer's Dement. 2015;11(3):332-84.

2. Yu JT, Tan L, Hardy J. Apolipoprotein E in Alzheimer's disease: an update. Annu Rev Neurosci. 2014:37:79-100.

3. Mattsson N, Andreasson U, Zetterberg H, Blennow K. Alzheimer's disease neuroimaging I: Association of Plasma Neurofilament Light with Neurodegeneration in patients with Alzheimer disease. JAMA Neurol. 2017;74(5):557-66.

4. Gaiottino J, Norgren N, Dobson R, Topping J, Nissim A, Malaspina A, Bestwick JP, Monsch AU, Regeniter A, Lindberg RL, et al. Increased neurofilament light chain blood levels in neurodegenerative neurological diseases. PLoS One. 2013;8(9):e75091.

5. Wang HF, Wan Y, Hao XK, Cao L, Zhu XC, Jiang T, Tan MS, Tan L, Zhang DQ, Tan $L$, et al. Bridging integrator 1 (BIN1) genotypes mediate Alzheimer's disease risk by altering neuronal degeneration. J Alzheimers Dis. 2016; 52(1):179-90

6. Chen J, Yu JT, Wojta K, Wang HF, Zetterberg H, Blennow K, Yokoyama JS, Weiner MW, Kramer JH, Rosen H, et al. Genome-wide association study identifies MAPT locus influencing human plasma tau levels. Neurology. 2017;88(7):669-76.

7. Kim S, Swaminathan S, Shen L, Risacher SL, Nho K, Foroud T, Shaw LM, Trojanowski JQ, Potkin SG, Huentelman MJ, et al. Genome-wide association study of CSF biomarkers Abeta1-42, t-tau, and p-tau181p in the ADNI cohort. Neurology. 2011;76(1):69-79.

8. Cruchaga C, Kauwe JS, Harari O, Jin SC, Cai Y, Karch CM, Benitez BA, Jeng AT, Skorupa T, Carrell D, et al. GWAS of cerebrospinal fluid tau levels identifies risk variants for Alzheimer's disease. Neuron. 2013;78(2):256-68.

9. Weiner MW, Veitch DP, Aisen PS, Beckett LA, Cairns NJ, Green RC, Harvey D, Jack CR, Jagust W, Liu E, et al. The Alzheimer's disease neuroimaging initiative: a review of papers published since its inception. Alzheimers Dement. 2013;9(5):e111-94.

10. Rohrer JD, Woollacott IO, Dick KM, Brotherhood E, Gordon E, Fellows A, Toombs J, Druyeh R, Cardoso MJ, Ourselin S, et al. Serum neurofilament light chain protein is a measure of disease intensity in frontotemporal dementia. Neurology. 2016;87(13):1329-36.

11. Desikan RS, Segonne F, Fischl B, Quinn BT, Dickerson BC, Blacker D, Buckner RL, Dale AM, Maguire RP, Hyman BT, et al. An automated labeling system for subdividing the human cerebral cortex on MRI scans into gyral based regions of interest. Neurolmage. 2006;31(3):968-80.

12. Jack CR Jr, Bernstein MA, Fox NC, Thompson P, Alexander G, Harvey D, Borowski B, Britson PJ, LW J, Ward C, et al. The Alzheimer's disease neuroimaging initiative (ADNI): MRI methods. J Magn Reson Imaging. 2008;27(4):685-91.

13. Lambert JC, Ibrahim-Verbaas CA, Harold D, Naj AC, Sims R, Bellenguez $C$, DeStafano AL, Bis JC, Beecham GW, Grenier-Boley B, et al. Meta-analysis of 
74,046 individuals identifies 11 new susceptibility loci for Alzheimer's disease. Nat Genet. 2013;45(12):1452-8.

14. Zetterberg H, Skillback T, Mattsson N, Trojanowski JQ, Portelius E, Shaw LM, Weiner MW, Blennow K. Alzheimer's disease neuroimaging I: association of cerebrospinal fluid Neurofilament light concentration with Alzheimer disease progression. JAMA Neurol. 2016;73(1):60-7.

15. Mattsson N, Insel PS, Palmqvist S, Portelius E, Zetterberg $H$, Weiner $M$, Blennow K, Hansson O. Alzheimer's disease neuroimaging I: cerebrospinal fluid tau, neurogranin, and neurofilament light in Alzheimer's disease. EMBO Mol Med. 2016;8(10):1184-96.

16. Olsson B, Lautner R, Andreasson U, Ohrfelt A, Portelius E, Bjerke M, Holtta M, Rosen C, Olsson C, Strobel G, et al. CSF and blood biomarkers for the diagnosis of Alzheimer's disease: a systematic review and meta-analysis. Lancet Neurol. 2016;15(7):673-84.

17. Fischbach BV, Trout KL, Lewis J, Luis CA, Sika M. WAGR syndrome: a clinical review of 54 cases. Pediatrics. 2005;116(4):984-8.

18. Kjeldsen E. A novel acquired cryptic three-way translocation $\mathrm{t}(2 ; 11 ; 5)$ (p21.3; q13.5; 23.2 ) with a submicroscopic deletion at 11p14.3 in an adult with hypereosinophilic syndrome. Exp Mol Pathol. 2015;99(1):50-5.

19. Zhao J, Zhao Y, Wang L, Zhang J, Karnes RJ, Kohli M, Wang G, Huang H. Alterations of androgen receptor-regulated enhancer RNAs (eRNAs) contribute to enzalutamide resistance in castration-resistant prostate cancer. Oncotarget. 2016;7(25):38551-65.

20. Wu M, Michaud EJ, Johnson DK. Cloning, functional study and comparative mapping of Luzp2 to mouse chromosome 7 and human chromosome 11p13-11p14. Mamm Genome. 2003;14(5):323-34.

21. Frisoni GB, Fox NC, Jack CR Jr, Scheltens P, Thompson PM. The clinical use of structural MRI in Alzheimer disease. Nat Rev Neurol. 2010;6(2):67-77.

22. Srivastava S, Cohen J, Pevsner J, Aradhya S, McKnight D, Butler E, Johnston M, Fatemi A. A novel variant in GABRB2 associated with intellectual disability and epilepsy. Am J Med Genet A. 2014;164A(11):2914-21.

23. Hirose S. Mutant GABA(a) receptor subunits in genetic (idiopathic) epilepsy. Prog Brain Res. 2014;213:55-85.

24. Ishii A, Kang JQ, Schornak CC, Hernandez CC, Shen W, Watkins JC, Macdonald RL, Hirose S. A de novo missense mutation of GABRB2 causes early myoclonic encephalopathy. J Med Genet. 2017;54(3):202-11.

25. Rissman RA, Mobley WC. Implications for treatment: GABAA receptors in aging, Down syndrome and Alzheimer's disease. J Neurochem. 2011; 117(4):613-22

\section{Ready to submit your research? Choose BMC and benefit from:}

- fast, convenient online submission

- thorough peer review by experienced researchers in your field

- rapid publication on acceptance

- support for research data, including large and complex data types

- gold Open Access which fosters wider collaboration and increased citations - maximum visibility for your research: over $100 \mathrm{M}$ website views per year 